\title{
Incidência e prevalência de dor fantasma em pacientes submetidos à amputação de membros: revisão de literatura
}

\author{
Prevalence of phantom pain in amputees: a systematic literature review
}

\author{
Danièlle Probstner ${ }^{1}$ e Luiz Claudio Santos Thuler ${ }^{2}$
}

\begin{abstract}
Resumo
Introdução: A maioria dos pacientes submetidos à amputação evolui com algum tipo de desconforto no membro ausente. Quando caracterizado como dor, esse desconforto é denominado dor fantasma, e reduz a qualidade de vida do paciente, que passa a solicitar de forma mais freqüente o sistema de saúde, inflacionando a demanda e trazendo implicações administrativas e de planejamento para a gestão da rede de atenção à saúde. Objetivo: Estudar a prevalência de dor fantasma em pacientes submetidos à amputação de membros, com ênfase naqueles com doença oncológica. Método: Foi realizada uma busca de estudos nas bases de dados Pubmed e Lilacs, no período de 01/01/2000 a 31/12/2005. Resultados: Foram identificados 11 estudos relacionados ao tema. Não foram encontrados trabalhos realizados na América Latina bem como aqueles compreendendo, exclusivamente, pacientes oncológicos. A prevalência de dor fantasma variou entre 26 e 80\% entre os estudos analisados. Conclusão: Poucos estudos abordaram a prevalência de dor fantasma no período considerado, sendo identificada uma carência de trabalhos sobre o tema, apontando a necessidade de pesquisas futuras sobre dor fantasma e suas peculiaridades em pacientes com câncer.
\end{abstract}

Palavras-Chave: Membro-fantasma, Neoplasias, Prevalência, Incidência.

\section{Abstract}

Introduction: Phantom sensation, painful or not, is usually present in the follow-up of subjects with an amputee. Phantom pain reduces the quality of life of patients, whom make heavy use of medical system. Because of this, the prevalence of phantom limb pain has an important role not only in the follow up of amputees, but in the management of medical system. Objectives: Systematic review of literature about phantom pain in amputees, taking into account oncologic pacients. Method: It was done a search at Internet data basis Pubmed and Lilacs from 2000/01/ 01 to 2005/12/31, looking for prevalence of phantom limb pain in amputees. It was also used the related article trick, available at Pubmed. Results: It was identified 11 studies related to prevalence, none of them were exclusively about oncologic patients. It was found a prevalence of phantom pain between $26 \%$ and $80 \%$. It was not found Latin Americans studies about prevalence of phantom limb pain in amputees. Conclusions: There were few studies about this theme in the last five years with a lack of Latin American data. Moreover, it was not found exclusive studies about oncologic patients, making possible future researches about phantom pain and its peculiarities in this kind of etiology.

Key words: Phantom Limb, Cancer, Prevalence, Incidence.

\footnotetext{
${ }^{1}$ Médica Ortopedista do Instituto Nacional de Câncer e Hospital de Traumato-Ortopedia, Mestranda do Curso de Neurologia da Universidade Federal do Estado do Rio de Janeiro (UNIRIO) - Rio de Janeiro - Brasil.

${ }^{2}$ Médico epidemiologista, Mestre em Ciências pela Universidade de Montreal, Doutor em Medicina pela Universidade Federal do Rio de Janeiro (UFRJ), Professor Adjunto da Universidade Federal do Estado do Rio de Janeiro (UNIRIO) e Médico Epidemiologista do Instituto Nacional de Câncer (INCA) - Rio de Janeiro - Brasil.

Programa de pós-graduação em Neurologia da Universidade Federal do Estado do Rio de Janeiro (UNIRIO) e Instituto Nacional de Câncer (INCA). Endereço para correspondência: Danièlle Probstner - Rua Duque de Caxias n ${ }^{\circ} 50$ apt $^{\circ} 201$, Vila Isabel CEP 20551-050, Rio de Janeiro - RJ. E-mail: dprobstner@oi.com.br
} 


\section{INTRODUÇÃO}

É sabido que a maioria dos pacientes submetidos à amputação evolui com algum tipo de desconforto no membro ausente ${ }^{1}$. Quando caracterizado como dor, esse desconforto reduz a qualidade de vida do paciente, que passa a utilizar de forma mais significativa o sistema de saúde, inflacionando a demanda, e trazendo, conseqüentemente, implicações administrativas e de planejamento para o gerenciamento da rede de atenção à saúde ${ }^{1}$. Vale lembrar que sensação fantasma, dor no coto e dor fantasma são entidades distintas, mas que podem coexistir num mesmo paciente, sendo fundamental distinguí-las semiologicamente para uma correta abordagem terapêutica ${ }^{1}$.

Credita-se, ao cirurgião militar francês Ambroise Paré (1510-1590), a primeira descrição médica a respeito de sensação fantasma dolorosa. Porém, o termo dor fantasma, de uso universal, só foi cunhado em 1871, por Silas Weir Mitchell1, ${ }^{2}$.

A dor fantasma pode se mostrar de caráter grave e de difícil controle, e deve ser diferenciada do quadro álgico que surge muitas vezes no coto de amputação, devido ao processo inflamatório inerente ao trauma cirúrgico ${ }^{1}$. Embora sua fisiopatologia ainda não esteja completamente estabelecida, estudos clínicos e experimentais vêm contribuindo de forma significativa para seu entendimento, havendo teorias centrais e periféricas para explicá-la ${ }^{1,3,4,5,6,7,8,9}$.

Além disso, são escassas as informaçōes na literatura científica sobre os fatores de risco associados à dor fantasma. Segundo Tomillero et al., poucos estudos epidemiológicos analisam esses fatores de forma global ou em seguimentos populacionais específicos ${ }^{6}$. Gallagher et al., ao estudarem dor fantasma em amputados de membro inferior, comentam que grande esforço tem sido feito no sentido de delinear fatores associados a esse fenômeno, destacando a dor no coto da amputação, a atividade física e a dor prévia à amputação ${ }^{7}$.

No passado, acreditava-se que a incidência da dor fantasma era baixa, em torno de $2 \%{ }^{1}$. Porém, estudos recentes contradizem estes dados, apresentando índices que variam de 60 a $80 \%^{1,2}$. Flor, ao discutir as características, causas e tratamento da dor fantasma, cita valores de prevalência que variam entre 50 e $80 \%$ com base em publicação de Jensen e Nikolajsen ${ }^{8}$. Já Manchikanti et al. fazem alusão a valores superiores a $80 \% \%^{4}$. Por sua vez, Nikolajsen et al., em artigo de revisão, sugerem que essas discrepâncias nas taxas de prevalência se devem ao fato dos estudos mais antigos basearem-se apenas em pacientes que buscaram tratamento para dor fantasma, subestimando assim sua real freqüência ${ }^{1}$. Já Borsje et al. realizaram uma análise de sensibilidade considerando diferentes critérios para definir dor fantasma e ponderaram que sua prevalência sofre consideráveis mudanças dependendo dos pontos de corte utilizados para definir o fenômeno ${ }^{10}$.

Nesse cenário, é compreensível que ainda não haja uma abordagem terapêutica padrão eficaz para a dor fantasma, uma vez que sua fisiopatologia não foi plenamente estabelecida. A abordagem terapêutica deste acometimento pode ser didaticamente organizada em três modalidades: medicamentosa, de apoio e cirúrgica, podendo ser utilizadas em conjunto ou separadamente ${ }^{1}$. No grupo medicamentoso de manuseio, tem sido utilizada uma vasta gama de drogas, como os antidepressivos tricíclicos, os bloqueadores de canal de sódio, anticonvulsivantes, anestésicos, calcitonina e menos eficazmente os opióides e antiinflamatórios não-esteróides (AINE) ${ }^{1}$. Por outro lado, os tratamentos de apoio incluem técnicas não-invasivas como estimulação elétrica nervosa transcutânea (TENS, do inglês transcutaneous electrical nerve stimulation), terapia vibratória, acupuntura, hipnose e biofeedback ${ }^{1}$. Abordagens cirúrgicas têm tido resultados insatisfatórios, sendo pouco utilizadas ${ }^{1}$.

Com base nos fatos expostos, foi realizada uma revisão sistemática de literatura sobre a prevalência de dor fantasma em pacientes submetidos à amputação de membros, buscando identificar esse índice naqueles com doença oncológica.

\section{MÉTODO}

Foi realizada uma revisão sistemática de literatura científica, referente ao período compreendido entre $01 / 01 / 2000$ e $31 / 12 / 2005$, nas bases de dados eletrônicas Pubmed e Lilacs. Utilizando-se simultâneamente as palavras-chave, em língua inglesa, phantom pain cancer prevalence or incidence, foram selecionados artigos que possuíam abstracts em língua inglesa, portuguesa ou espanhola. Não houve limite quanto à faixa etária.

A pesquisa identificou oito artigos no Pubmed e nenhum no Lilacs. Desses, apenas um era referente ao objeto desse estudo. A partir deste trabalho, utilizou-se o aplicativo related articles, disponível no Pubmed, com o objetivo de encontrar artigos afins. Esse procedimento foi realizado para cada novo artigo identificado, até que os resultados da busca tornaram-se repetitivos, identificando-se sempre os mesmos trabalhos.

Foram incluídos nesta revisão os artigos que possuíam casuística própria e que abordavam de forma direta ou indireta a prevalência ou a incidência de dor fantasma em pacientes submetidos à amputação de membros.

Os estudos obtidos foram analisados quanto ao seu 
desenho, número de pacientes avaliados, distribuição quanto ao sexo do paciente, local da amputação, instrumentos utilizados para a coleta de dados e para a medida da dor, sua forma de aplicação (auto-aplicados ou não), veículo utilizado para sua aplicação (via postal ou entrevistador), tempo após a amputação em que foi realizada a avaliação, prevalência ou incidência de dor fantasma e doença de base que levou à amputação.

\section{RESULTADOS}

Entre os 11 trabalhos incluídos nesta revisão da literatura, não foram identificados trabalhos realizados exclusivamente em pacientes oncológicos ou referentes a pacientes latino-americanos.

Conforme apresentado na tabela 1 , oito estudos eram transversais ${ }^{7,10,11,12,13,14,15,16}$, enquanto apenas três eram prospectivos $^{6,17,18}$. O número de pacientes avaliados variou de 39 a 914. A maioria dos pacientes avaliados teve, como doença de base, o trauma, cuja freqüência variou de 23,5 a $100,0 \%$ dos casos. Nesses estudos, o percentual de pacientes com doença oncológica esteve entre 0 e $23,7 \%$.

Os dados apresentados na tabela 2 mostram que houve grande disparidade na prevalência apresentada de dor fantasma, nos diferentes trabalhos, com variaçôes entre 26 e $80 \%$. Além disso, foi constatada a inexistência de padronização dos instrumentos para a coleta dos

Tabela 1. Principais características dos estudos de prevalência de dor fantasma (PARTE I)

\begin{tabular}{|c|c|c|c|c|c|c|}
\hline Autor & $\begin{array}{c}\text { Ano da } \\
\text { publicação }\end{array}$ & $\begin{array}{l}\text { Desenho do } \\
\text { estudo }\end{array}$ & $\begin{array}{l}\text { Número de } \\
\text { pacientes }\end{array}$ & $\begin{array}{l}\text { Local da } \\
\text { amputação }\end{array}$ & $\begin{array}{c}\text { Tempo } \\
\text { desde a } \\
\text { amputação }\end{array}$ & $\begin{array}{l}\text { Etiologia da } \\
\text { amputação }\end{array}$ \\
\hline $\begin{array}{l}\text { Kooijman } \\
\text { et al. }{ }^{11}\end{array}$ & 2000 & transversal & 99 & $M S=100,0 \%$ & ND & $\begin{array}{c}\text { trauma }=78,0 \% \\
\text { câncer }=15,0 \% \\
\text { vascular }=3,0 \%\end{array}$ \\
\hline Ehde et al. ${ }^{13}$ & 2000 & transversal & 225 & $M I=100,0 \%$ & 6 meses & $\begin{array}{c}\text { trauma }=23,5 \% \\
\text { infecção }=10,2 \% \\
\text { câncer }=0,8 \%\end{array}$ \\
\hline $\begin{array}{l}\text { Gallagher et } \\
\text { al. }^{7}\end{array}$ & 2001 & transversal & 104 & $M I=100,0 \%$ & ND & $\begin{array}{l}\text { trauma }=49 \% \\
\text { câncer }=23,1 \% \\
\text { outras }=19,3 \%\end{array}$ \\
\hline Whyte et al. ${ }^{18}$ & 2001 & $\begin{array}{c}\text { coorte } \\
\text { prospectivo }\end{array}$ & 89 & $M I=100,0 \%$ & ND & ND \\
\hline $\begin{array}{l}\text { Dijkstra } \\
\text { et al. }{ }^{12}\end{array}$ & 2002 & transversal & 536 & $\begin{array}{l}M I=81,0 \% \\
M S=19,0 \%\end{array}$ & 18,8 anos & $\begin{array}{c}\text { trauma }=38,0 \% \\
\text { vascular }=23,7 \% \\
\text { câncer }=9,1 \%\end{array}$ \\
\hline $\begin{array}{l}\text { Lacoux } \\
\text { et al. }{ }^{14}\end{array}$ & 2002 & transversal & 40 & $M S=100,0 \%$ & 22 meses & trauma $=100 \%$ \\
\hline $\begin{array}{l}\text { van der Schans } \\
\text { et al. }{ }^{16}\end{array}$ & 2002 & transversal & 437 & $M I=100,0 \%$ & 10 anos & $\begin{array}{c}\text { trauma }=34 \% \\
\text { vascular }=29 \% \\
\text { câncer }=9,0 \%\end{array}$ \\
\hline $\begin{array}{l}\text { Tomillero } \\
\text { et al. }{ }^{6}\end{array}$ & 2002 & $\begin{array}{c}\text { coorte } \\
\text { prospectivo }\end{array}$ & 53 & $M I=100,0 \%$ & 5 dias & vascular $=100 \%$ \\
\hline Borsje et al. ${ }^{10}$ & 2004 & transversal & 536 & $\begin{array}{l}M I=81,5 \% \\
M S=18,5 \%\end{array}$ & ND & $\begin{array}{c}\text { trauma }=38,4 \% \\
\text { vascular }=23,7 \% \\
\text { câncer }=9,1 \%\end{array}$ \\
\hline $\begin{array}{l}\text { Stremmel } \\
\text { et al. }{ }^{17}\end{array}$ & 2005 & $\begin{array}{c}\text { coorte } \\
\text { prospectivo }\end{array}$ & 39 & $M I=100,0 \%$ & ND & vascular $=100 \%$ \\
\hline $\begin{array}{l}\text { Ephraim } \\
\text { et al. }{ }^{15}\end{array}$ & 2005 & transversal & 914 & $\begin{array}{l}M I=88,8 \% \\
M S=10,9 \%\end{array}$ & 4 anos & $\begin{array}{c}\text { trauma }=39 \% \\
\text { vascular }=37,1 \% \\
\text { câncer }=23,7 \%\end{array}$ \\
\hline
\end{tabular}

Abreviaçōes: $\mathrm{ND}=$ não disponível; $\mathrm{MS}=$ membros superiores; $\mathrm{MI}=$ membros inferiores 
Tabela 2. Principais características dos estudos de prevalência de dor fantasma (PARTE II)

\begin{tabular}{|c|c|c|c|c|c|c|}
\hline Autor & $\begin{array}{c}\text { Ano da } \\
\text { publicação }\end{array}$ & $\begin{array}{l}\text { Instrumento de } \\
\text { medida }\end{array}$ & $\begin{array}{c}\text { Escala } \\
\text { específica de } \\
\text { dor utilizada }\end{array}$ & $\begin{array}{c}\text { Forma de aplicação } \\
\text { do questionário }\end{array}$ & $\begin{array}{l}\% \text { de } \\
\text { resposta }\end{array}$ & $\begin{array}{c}\text { Prevalênci a } \\
\text { de dor } \\
\text { fantasma }\end{array}$ \\
\hline $\begin{array}{l}\text { Kooijman } \\
\text { et al. }{ }^{11}\end{array}$ & 2000 & GQPAA & nenhuma & $\begin{array}{l}\text { auto-aplicado por } \\
\text { via postal }\end{array}$ & $80,0 \%$ & $51,0 \%$ \\
\hline Ehde et al. ${ }^{13}$ & 2000 & $\begin{array}{l}\text { Questionário } \\
\text { próprio }\end{array}$ & $\mathrm{EVN} / \mathrm{CPG}$ & $\begin{array}{l}\text { auto-aplicado por } \\
\text { via postal }\end{array}$ & $56,0 \%$ & $72,0 \%$ \\
\hline $\begin{array}{l}\text { Gallagher } \\
\text { et al. }{ }^{7}\end{array}$ & 2001 & TAPES & nenhuma & $\begin{array}{l}\text { aplicado por } \\
\text { entrevistador }\end{array}$ & $100,0 \%$ & $69,2 \%$ \\
\hline Whyte et al. ${ }^{18}$ & 2001 & $\begin{array}{l}\text { Questionário } \\
\text { próprio }\end{array}$ & EVN & $\begin{array}{l}\text { auto-aplicado por } \\
\text { via postal }\end{array}$ & $89,0 \%$ & $71,0 \% *$ \\
\hline Dijkstra et al. ${ }^{12}$ & 2002 & $\begin{array}{c}\text { GQPAA + } \\
\text { GQPLA }\end{array}$ & nenhuma & $\begin{array}{l}\text { auto-aplicado por } \\
\text { via postal }\end{array}$ & $34,0 \%$ & $72,0 \%$ \\
\hline Lacoux et al. ${ }^{14}$ & 2002 & $\begin{array}{l}\text { Questionário } \\
\text { próprio }\end{array}$ & EVN & $\begin{array}{l}\text { aplicado por } \\
\text { entrevistador }\end{array}$ & $100,0 \%$ & $29,0 \%$ \\
\hline $\begin{array}{l}\text { van der Schans } \\
\text { et al. }{ }^{16}\end{array}$ & 2002 & $\begin{array}{c}\text { GQPLA + } \\
\text { RAND36DLV }\end{array}$ & nenhuma & $\begin{array}{l}\text { auto-aplicado por } \\
\text { via postal }\end{array}$ & $82,0 \%$ & $80,0 \%$ \\
\hline $\begin{array}{l}\text { Tomillero } \\
\text { et al. }{ }^{6}\end{array}$ & 2002 & $\begin{array}{l}\text { Questionário } \\
\text { próprio }\end{array}$ & EVN & $\begin{array}{l}\text { aplicado por } \\
\text { entrevistador }\end{array}$ & $100,0 \%$ & $26,0 \% *$ \\
\hline Borsje et al. ${ }^{10}$ & 2004 & $\begin{array}{l}\text { GQPLA + } \\
\text { GQPAA }\end{array}$ & nenhuma & $\begin{array}{l}\text { auto-aplicado por } \\
\text { via postal }\end{array}$ & $34,4 \%$ & $9,0-72,0 \%$ \\
\hline $\begin{array}{l}\text { Stremmel } \\
\text { et al. }{ }^{17}\end{array}$ & 2005 & $\begin{array}{l}\text { Questionário } \\
\text { próprio }\end{array}$ & nenhuma & $\begin{array}{l}\text { aplicado por } \\
\text { entrevistador }\end{array}$ & $100,0 \%$ & $69,2 \% *$ \\
\hline $\begin{array}{l}\text { Ephraim } \\
\text { et al. }{ }^{15}\end{array}$ & 2005 & $\begin{array}{l}\text { Questionário } \\
\text { próprio }\end{array}$ & EVN & aplicado via telefone & $59,4 \%$ & $80,0 \%$ \\
\hline
\end{tabular}

dados e para a medida de dor. Dos 11 estudos analisados, três utilizaram o Groningen Questionnaire Problems after Leg Amputation (GQPLA) ${ }^{10,12,16}$, associado ou não ao Groningen Questionnaire Problems after Arm Amputation (GQPAA) ${ }^{10,11,12}$. Por outro lado, um estudo descritivo de dor fantasma em pacientes com amputação de membro inferior empregou o Trinity Amputation and Prosthesis Experience Scales (TAPES) 7 e os demais estudos fizeram uso de questionários elaborados pelos próprios autores, baseados ou não em instrumentos já existentes como o questionário de dor McGill simplificado. Em um estudo ${ }^{16}$, foi utilizado junto com o GQPLA um instrumento para avaliação global da saúde, o RAND36 DVL (versão holandesa do questionário de qualidade de vida utilizado pelo Medical Outcome Study, o Short Form 36 - SF36).

Dos artigos de prevalência encontrados, 54,5\% utilizaram instrumentos de coleta de dados autoaplicados, via correio ${ }^{10,11,12,13,16,18}$, enquanto que, em
$45,5 \%$, a aplicação destes foi feita pessoalmente por intermédio de entrevistadores $6,7,14,15,17$. Houve um único estudo no qual o instrumento foi aplicado por telefone, após o recebimento de uma carta previamente enviada solicitando o consentimento ${ }^{15}$.

\section{DISCUSSÃO}

Embora dor fantasma seja um tema muito discutido na literatura científica, nos últimos anos, estudos com enfoque em sua prevalência em pacientes amputados são escassos e apresentam grande variabilidade nos resultados ${ }^{11,12,14}$. Neste estudo, observou-se que a prevalência de dor fantasma variou entre 26 e $80 \%$. Essa variação já havia sido indicada por outros autores que apontam como principais fatores: a falta de conhecimento sobre o assunto por parte dos pacientes e médicos assistentes, a falta de homogeneização de critérios e instrumentos de coleta de dados que 
caracterizam o fenômeno e as diferentes respostas terapêuticas ${ }^{1,2,9}$. Além disso, instrumentos auto-aplicados, recebidos em domicílio pelo correio, apresentam índices substanciais de não-resposta ${ }^{9,11,12,13,16,18}$, o que pode comprometer os índices de prevalência obtidos, constituindo-se num viés de seleção.

Durante a análise dos textos, fica claro que não há padronização no emprego de instrumentos para investigar e medir a dor. Esta avaliação, de uma maneira geral, é feita através de perguntas a respeito das características da dor em diferentes tipos de questionários criados para analisar este acometimento. A falta de padronização torna as informações heterogêneas, o que, por sua vez, dificulta a análise comparativa das mesmas.

Outro ponto importante a ser observado é que a dor prévia à amputação tem sido apontada como fator de risco para o desencadeamento de dor fantasma ${ }^{1}$, o que justificaria um estudo exclusivo da prevalência deste fenômeno em patologias, cujo quadro álgico é um aspecto clínico usual. Tal pesquisa poderia averiguar se o índice de prevalência de dor fantasma observado nos estudos atuais pode ser estendido a grupos de pacientes com etiologias específicas que apresentam quadro álgico prévio freqüente, como ocorre, por exemplo, nos pacientes com câncer.

Por vezes, observa-se confusão com relação ao emprego correto da terminologia das medidas de freqüência avaliadas, falando-se ora de incidência ora de prevalência. Por exemplo, em um estudo de coorte prospectivo $^{6}$, o autor refere-se à prevalência de dor fantasma, embora, ao que tudo indica, se trate de casos novos (incidentes). Com relação aos estudos sobre fatores de risco para dor fantasma, são poucos aqueles que apresentam análises bivariadas e multivariadas ${ }^{6,17,18}$. A carência destas informações traz pouca consistência para a avaliação desses fatores.

Conclui-se que os estudos que abordam a prevalência de dor fantasma são escassos, não sendo encontradas informaçōes referentes aos países da América Latina no período estudado. Além disso, não foram identificadas pesquisas exclusivas sobre o tema em pacientes com doença oncológica, o que sugere que a realização de futuros estudos sobre o assunto poderia trazer esclarecimentos sobre as particularidades de sua ocorrência neste grupo populacional e, conseqüentemente, aos desdobramentos dela advindos. Além disso, a inexistência de estudos envolvendo exclusivamente pacientes com câncer constitui-se em uma lacuna no conhecimento científico, visto que se trata de importante causa de morbi-mortalidade em nosso meio.

\section{AGRADECIMENTOS}

À Neli Muraki Ishikawa pelas valiosas críticas e sugestôes apresentadas ao texto.

\section{REFERÊNCIAS}

1. Nikolajsen L, Jensen TS. Phantom limb pain. Br J Anaesth. 2001; 87:107-16.

2. Woodhouse A. Phantom limb sensation. Clin Exp Pharmacol Physiol. 2005;32:132-4.

3. Dettmers C, Adler T, Rzanny R, Van Schayck R, Gaser C, Weiss $\mathrm{T}$, et al. Increased excitability in the primary motor cortex and supplementary motor area in patients with phantom limb pain after upper limb amputation. Neuroscience Lett. 2001;307:109-12.

4. Manchikanti L, Singh V. Managing phantom pain. Pain Physician. 2004; 7:365-75

5. Melzack R. Phantom limbs and the concept of neuromatrix. TINS. 1990;13(3):88-92

6. Tomillero FA, de la Torre RR, Barrientos FC, Torrejón AH, Moreno ME, García AG, et al. Estudio prospectivo de la prevalencia y factores de riesgo de miembro fantasma doloroso en el postoperatorio inmediato de pacientes sometidos a amputación por isquemia arterial crónica. Rev Esp Anestesiol Reanim. 2002;49:295-301

7. Gallagher P, Allen D, MacLachlan M. Phantom limb pain and residual limb pain following lower limb amputation : a descriptive analysis. Disabil Rehabil. 2001;23(12):522-30.

8. Flor H. Phantom -limb pain: characteristics, causes, and treatment. Lancet Neurol. 2002;1:182-9

9. Flor H, Denke C, Schaefer M, Grüsser S. Effect of sensory discrimination training on cortical reorganization and phantom limb pain. Lancet North Am Ed. 2001 June;357(2):1763-4

10. Borsje S, Bosmans JC, van der Schans CP, Geertzen JHB, Dijkstra PU. Phantom pain : a sensitivity analysis. Disabil Rehabil. 2004;26(14-15): 905-10

11. Kooijman CM, Dijkstra PU, Geertzen JHB, Elzinga A, Van der Schans CP. Phantom pain and phantom sensations in upper limb amputes: an epidemiological study. Pain. 2000;87:33-41

12. Dijkstra PU, Geertzen JHB, Stewart R, Van der Schans CP. Phantom pain and risk factors: a multivariate analysis. J Pain Symptom Manage. 2002 December;24(6):578-85

13. Ehde DM, Czerniecki JM, Smith DG, Campbell KM, Edwards T, Jensen MP, et al. Chronic phantom sensations, phantom pain, residual limb pain and other regional pain after lower limb amputation. Arch Phys Med Reabil. 2000;81:1039-44

14. Lacoux PA, Crombie IK, Macrae WA. Pain in traumatic upper limb amputees in Sierra Leone. Pain.2002;99(12):309-12 
15. Ephraim PL, Wegener ST, MacKenzie EJ, Dillingham TR, Pessin LE. Phantom pain, residual limb pain, and back pain in amputees : results of a national survey. Arch Phys Med Rehabil.2005;86:1911-9

16. van der Schans CP, Geertzen JHB, Schoppen T, Dijkstra PU. Phantom pain and health-related quality of life in lower limb amputees. J Pain Symptom Manage.2002;24:429-36
17. Stremmel C, Horn C, Eder S, Dimmler A, Lang W. The impact of immunological parameters on the development of phantom pain after major amputation. Eur J Endovasc Surg. 2005;30:79-82.

18. Whyte AS, Niven CA. Variation in phantom limb pain : results of a diary study. J Pain Symptom Manage. 2001;22:947-53 